\title{
APPROXIMATION ON DISKS
}

\author{
P. J. DE PAEPE
}

\begin{abstract}
It is shown that if the functions $F$ and $G$ are defined in a neighborhood of the origin in the complex plane and are in a certain sense like $z^{m}$ and $\bar{z}^{n}$ with $\operatorname{gcd}(m, n)=1$, then on sufficiently small closed disks $D$ around 0 every continuous function on $D$ can be uniformly approximated by polynomials in $F$ and $G$.
\end{abstract}

In the following we are interested in uniform approximation on compact subsets of the complex plane $\mathbf{C}$. If $f_{1}, \ldots, f_{n}$ are continuous functions on such a compact set $X$, separating the points of $X,\left[f_{1}, \ldots, f_{n} ; X\right]$ denotes the function algebra on $X$ generated by $f_{1}, \ldots, f_{n}$, i.e. the uniform closure in $C(X)$ of polynomials in $f_{1}, \ldots, f_{n}$. Much is known for the situation $A=[z, f ; D]$ where $D$ denotes a closed disk in $\mathbf{C}$, see for instance $[\mathbf{1}, \mathbf{2}, \mathbf{4}-\mathbf{7}$ and 9]. A possible way to generalize is to replace $z$ by another function. Minsker [3] considered the case $A=\left[z^{m}, \bar{z}^{n} ; X\right]$ where $\operatorname{gcd}(m, n)=1$. He shows $A=C(D)$ by proving $z$ and $\bar{z}$ belong to $A$ so that the classical Stone-Weierstrass theorem can be applied. Essential in his proof is the representation of the functions $z$ and $\bar{z}$ as a quotient of elements of $A$. In [6] a very short proof of Minsker's result is given based on the Bishop-Stone-Weierstrass theorem on maximal sets of antisymmetry (see for instance [8]). In this note we give a local generalization of the theorem of Minsker.

THEOREM. Let $F(z)=z^{m}(1+f(z)), G(z)=\bar{z}^{n}(1+g(z))$ where $f$ and $g$ are functions defined in a neighborhood of 0 , of class $C^{1}$, with $f(0)=0, g(0)=0$. If $\operatorname{gcd}(m, n)=1$ and if $D$ is a sufficiently small closed disk around 0 then $[F, G ; D]=$ $C(D)$.

The proof consists of appropriate generalizations of the essential steps in Minsker's proof for the case $F(z)=z^{m}, G(z)=\bar{z}^{n}$. We start with a simple lemma on quotients of elements in a function algebra.

LEMMA. Let $A$ be a function algebra on a compact Hausdorff space $X$, let $f, g \in$ $A$ such that $\{g=0\} \subset\{f=0\}$ and such that $f / g$, defined to be 0 at $\{g=0\}$, is continuous on $X$. Suppose there is a sequence $\left\{\alpha_{n}\right\}_{n}$ of complex numbers with $\lim \alpha_{n}=0$ such that for each $n$ the function $g-\alpha_{n}$ is invertible in $A$ and such that $\left\{\alpha_{n}\left(g-\alpha_{n}\right)^{-1}\right\}_{n}$ is a bounded sequence in $A$. Then $f / g \in A$.

PROOF. Let $\varepsilon>0$ and let $M>0$ be an upperbound on the supremum norms of the elements of $\left\{\alpha_{n}\left(g-\alpha_{n}\right)^{-1}\right\}_{n}$. Let $h_{n}=f \cdot\left(g-\alpha_{n}\right)^{-1}$. Then $h_{n} \in A$ and for $x \in X$

$$
\left|(f / g)(x)-h_{n}(x)\right|=\left|\alpha_{n}(f / g)(x) \cdot\left(g(x)-\alpha_{n}\right)^{-1}\right| .
$$

Received by the editors July 31, 1984 and, in revised form, June 20, 1985.

1980 Mathematics Subject Classification. Primary $46 \mathrm{~J} 10$.

Key words and phrases. Function algebra, Stone-Weierstrass theorem, uniform approximation in the complex plane. 
If $|(f / g)(x)|<\varepsilon / M$, then clearly $\left|(f / g)(x)-h_{n}(x)\right|<\varepsilon$. On $\{|f / g| \geq \varepsilon / M\}, g$ has no zeros, so $\left|g-\alpha_{n}\right|$ is bounded away from zero because $\lim \alpha_{n}=0$, so there is a $\delta>0$ with $\left|g(x)-\alpha_{n}\right| \geq \delta$ for all $n$ and all $x$ with $|(f / g)(x)| \geq \varepsilon / M$. For such $x$

$$
\left|(f / g)(x)-h_{n}(x)\right| \leq\left|\alpha_{n}\right| \cdot\|f / g\| / \delta
$$

Hence for $n$ sufficiently large $\left\|f / g-h_{n}\right\|<\varepsilon$. So $f / g \in A$.

REMARK. Note that, if moreover, $g$ belongs to a closed ideal $I$ of $A$, then $f / g$ also belongs to $I$ since $f / g$ is the uniform limit on $X$ of the sequence $\left\{g \cdot(f / g) \cdot\left(g-\alpha_{n}\right)^{-1}\right\}_{n}$ in $I$.

Corollary 1. Let $A$ be a function algebra on $X$, let $f, g \in A$ such that $\{g=$ $0\} \subset\{f=0\}$ and such that $f / g$, defined to be 0 at $\{g=0\}$, is continuous on $X$. Suppose $\operatorname{Re} g \geq 0$ on $X$. Then $f / g \in A$.

PROOF. Take $\alpha_{n}=-1 / n$ in the lemma.

Corollary 1 will be an essential ingredient in the proof of the theorem. The shortest way to finish the proof of this theorem is to use a recent result of O'Farrell and Preskenis [5] which states that if $f$ and $g$ are $C^{\mathbf{1}}$ diffeomorphisms of a closed disk $D$ into $\mathbf{C}$, having opposite degrees, then $[f, g ; D]=C(D)$. But we only need a local form of their result which can be seen as a consequence of a combination of an old theorem of Wermer [9] and a result on homomorphism spaces of certain function algebras. This last result occurs in one form or another in many of the papers dealing with the determination of algebras of the type $[z, f ; D]$. Although there exists a stronger result (indeed, the Lipschitz-condition on $f$ can be disposed of, see the proof of Theorem (3.2) in [4]) we like to give a short proof for this special case, based on Corollary 1 . We denote the complex homomorphism space of a function algebra $A$ by $\Delta A$.

Corollary 2. Let $D$ be a closed disk in $\mathbf{C}$, let $f \in \operatorname{Lip}(\alpha, D)$ for some $\alpha>0$, i.e. there is a constant $k$ with $|f(z)-f(a)| \leq k|z-a|^{\alpha}$ for all $z, a \in D$. Suppose $\operatorname{Re}(z-a)(f(z)-f(a)) \geq 0$ for all $z, a \in D$. Then $\Delta[z, f ; D]=D$.

Proof. Abbreviate $A=[z, f ; D]$ and let $N \in \mathbf{N}$ with $N \alpha>1$. Let $\phi \in \Delta A$. If $a=\phi(z)$, then $a \in D$. Since $(f-f(a))^{N+1} /(z-a)(f-f(a))$, i.e. $(f-f(a))^{N} /(z-a)$ is continuous on $D$, we can apply Corollary 1 and conclude that $(f-f(a))^{N} /(z-a) \in$ $A$. Hence

$$
\begin{aligned}
(\phi(f)-f(a))^{N} & =\phi\left((f-f(a))^{N}\right)=\phi(z-a) \cdot \phi\left((f-f(a))^{N} /(z-a)\right) \\
& =0 \cdot \phi\left((f-f(a))^{N} /(z-a)\right)=0 .
\end{aligned}
$$

So $\phi(f)=f(a)$ which, together with $\phi(z)=a$, implies $\phi(g)=g(a)$ for all $g \in A$. So $\phi$ is point evaluation at some point of $D$.

The theorem of Wermer reads as follows:

Let $D$ be a closed disk centered at the origin and let $f$ be defined in a neighborhood of $D$, of class $C^{1}$. Suppose $f_{\bar{z}}(a) \neq 0$ for all $a \in D$. If $\Delta[z, f ; D]=D$, then $[z, f ; D]=C(D)$.

Proposition. Let $f$ be defined in a neighborhood of 0 , of class $C^{1}$, such that $f_{\bar{z}}(0) \neq 0$. Then $[z, f ; D]=C(D)$ if $D$ is a sufficiently small closed disk around 0 . 
ProOF. Observe that we may assume $f_{z}(0)=0, f_{\bar{z}}(0)=1$ (replace $f$ by $\left.f_{\bar{z}}(0)^{-1} \cdot\left(f-f_{z}(0) z\right)\right)$. Since the first order partial derivatives of $f$ are continuous near 0 Taylor's formula can be applied to $\operatorname{Re} f$ and $\operatorname{Im} f$ to get a $\delta>0$ such that

$f$ is defined on $D=\{|z| \leq \delta\}$,

$f_{\bar{z}}$ has no zeroes on $D$,

$\left|f(z)-f(a)-f_{z}(0)(z-a)-f_{\bar{z}}(0)(\bar{z}-\bar{a})\right|=|f(z)-f(a)-(\bar{z}-\bar{a})| \leq|z-a|$ for $z, a \in D$.

(O'Farrell and Preskenis use Taylor's formula in the proof of Lemma 1 in [5] in a similar way.) Hence $|(z-a)(f(z)-f(a))-| z-\left.a\right|^{2}|\leq| z-\left.a\right|^{2}$ for $z, a \in D$, so $\operatorname{Re}(z-a)(f(z)-f(a)) \geq 0$ for $z, a \in D$. By Corollary $2 \Delta[z, f ; D]=D$, hence by Wermer's theorem $[z, f ; D]=C(D)$.

PROOF OF THE THEOREM. Let $D$ be a closed disk centered at 0 in the domain of definition of $f$ and $g$ such that $|f(z)|<1,|g(z)|<1$ for all $z \in D$. Let for $k \in \mathbf{N} h_{k}(w)$ be the branch of the $k$ th root of $w$ defined on $\{\operatorname{Re} w \geq 0\}$ with $h_{k}(1)=1$. Define $p(z)=z h_{m}(1+f(z)), q(z)=\bar{z} h_{n}(1+g(z))$. Then $p$ and $q$ are $C^{1}$ functions in a neighborhood of $D$. Moreover, $p_{z}(0)=1, p_{\bar{z}}(0)=0$ and $q_{z}(0)=0, q_{\bar{z}}(0)=1$ and if $D$ is sufficiently small, $p$ and $q$ are homeomorphisms from $D$ into $\mathbf{C}$.

Of course, $p^{m}=F, q^{n}=G$ on $D$, so we also have $(p q)^{m n}=F^{n} G^{m}$. Now for $z \in D,(p(z) q(z))^{m n}=|z|^{2 m n}(1+f(z))^{n}(1+g(z))^{m}$, so $\operatorname{Re}(p q)^{m n} \geq 0$ on small disks $D$. On compact subsets of $\{\operatorname{Re} w \geq 0\}$ the function $h_{m n}$ can be uniformly approximated by polynomials. Hence $h_{m n}\left(F^{n} G^{m}\right)$ can be uniformly approximated on $D$ by polynomials in $F$ and $G$ and for small disks $D$ this function is equal to $p q$. Let $a \in \mathbf{N}, b \in \mathbf{N} \cup\{0\}$ with $a m-b n=\mathbf{1}$ and write in a way analogous to Minsker

$$
p=p^{a m-b n}=\left(p^{m}\right)^{a} /\left(p^{n}\right)^{b}=\left(p^{m}\right)^{a}\left(q^{n}\right)^{b} /(p q)^{n b}=F^{a} G^{b} /(p q)^{n b} .
$$

From this it follows that the functions $F$ and $G$ separate the points of $D$ since it is easily seen that $p^{m}, q^{n}$ and $p q$ do so. Let $A=[F, G ; D]$. Since the only zero of $p q$ in $D$ is at 0 , it follows by repeated use of Corollary 1 (dividing $n b$ times by $p q$ ) that $p \in A$. In a similar way it follows that $q \in A$. Therefore $A=[p, q ; D]$.

Now since $\left(q \circ p^{-1}\right)_{\bar{z}}(0)=1$ we can apply the proposition to obtain $\left[z, q \circ p^{-1} ; E\right]=C(E)$ for sufficiently small disks $E$ around the origin. But then of course $[p, q ; D]=C(D)$ if $D$ is sufficiently small. This shows that $[F, G ; D]=C(D)$ if $D$ is small enough.

REMARK. Suppose that $F$ and $G$ separate points near 0 , but instead of $\operatorname{gcd}(m, n)$ $=1$ we have $\operatorname{gcd}(m, n)=k$ with $k>1$. Then with notation as above we obtain $[F, G ; D]=\left[p^{k}, q^{k} ; D\right]$ for sufficiently small disks $D$. Hence the problem of determining $[F, G ; D]$ for small disks reduces to the case where $F$ and $G$ have zeroes at the origin of the same order. An open problem is to determine the algebra $\left[z^{2}, \bar{z}^{2}+\bar{z}^{3} ; D\right]$ for small $D$ around 0 .

EXAMPLE 1. Let $F$ and $G$ be analytic at 0 with Taylor series expansions $z^{m}+$ $a_{m+1} z^{m+1}+\cdots$, respectively $z^{n}+b_{n+1} z^{n+1}+\cdots, \operatorname{gcd}(m, n)=1$. Then, for sufficiently small $D$ we have $[F, \bar{G} ; D]=C(D)$.

REMARK. The conditions on $f$ and $g$ in the theorem can be weakened. To obtain that $p$ and $q$ in the proof of the theorem are $C^{1}$ functions it suffices that $f$ and $g$ are continuous functions defined in a neighborhood of 0 , with $f(0)=0$, $g(0)=0$ and with continuous and bounded partial derivatives of first order outside the origin. 
EXAMPLE 2. The theorem can now also be applied to the functions $F(z)=z$ and $G(z)=\bar{z}^{2}\left(1+z^{2} \bar{z}^{-1}\right)$ and we obtain that for small $D\left[z, \bar{z}^{2}+z^{2} \bar{z} ; D\right]=C(D)$. Observe that this problem was left open in [1].

\section{REFERENCES}

1. M. Freeman, The polynomial hull of a thin two-manifold, Pacific J. Math. 38 (1971), 377-389.

2. S. N. Mergelyan, Uniform approximations to functions of a complex variable, Amer. Math. Soc. Transl. (1) 3 (1962), 281-391.

3. S. Minsker, Some applications of the Stone-Weierstrass theorem to planar rational approximation, Proc. Amer. Math. Soc. 58 (1976), 94-96.

4. A. G. O'Farrell and K. J. Preskenis, Approximation by polynomials in two complex variables, Math. Ann. 246 (1980), 225-232.

5. , Approximation by polynomials in two diffeomorphisms, Bull. Amer. Math. Soc. (N.S.) 10 (1984), 105-107.

6. P. J. de Paepe, Some applications of the Stone-Weierstrass theorem, Proc. Amer. Math. Soc. 70 (1978), 63-66.

7. K. J. Preskenis, Approximation on disks, Trans. Amer. Math. Soc. 171 (1972), 445-467.

8. E. L. Stout, The theory of uniform algebras, Bogden and Quigley, Tarrytown-on-Hudson, N. Y., 1971.

9. J. Wermer, Polynomially convex disks, Math. Ann. 158 (1965), 6-10.

DePaRTMENT OF MATHEMATICS, UNiVERSity OF AMSTERdaM, ROETERSTRAat 15, 1018 WB AMSTERDAM, THE NETHERLANDS 